\title{
450.
}

\section{NOTE ON THE THEORY OF THE RATIONAL TRANSFORMATION BETWEEN TWO PLANES, AND ON SPECIAL SYSTEMS OF POINTS.}

[From the Proceedings of the London Mathematical Society, vol. III. (1869-1871), pp. 196-198. Read December 8, 1870.]

IN Prof. Cremona's theory of the transformation of plane curves, the fundamental equations are taken to be

$$
\begin{aligned}
& \alpha_{1}+4 \alpha_{2}+9 \alpha_{3}+\ldots=n^{2}-1 \\
& \alpha_{1}+3 \alpha_{2}+6 \alpha_{3}+\ldots=\frac{1}{2}\left(n^{2}+3 n\right)-2
\end{aligned}
$$

and from these we have as a consequence

$$
\alpha_{2}+3 \alpha_{3}+\ldots=\frac{1}{2}(n-1)(n-1)
$$

viz., the first equation expresses that any two curves of the system intersect in a single variable point; the second, that the curves form a réseau, or system containing two arbitrary parameters; and the third, that the curves are unicursal.

In the equivalent theory of the rational transformation between two planes, as given in my "Memoir on the Rational Transformation between Two Spaces," [447], we have the equation (1); but instead of the equation (2), it would primâ facie appear to be sufficient if we had the inequation

$$
\alpha_{1}+3 \alpha_{2}+6 \alpha_{3}+\ldots<\frac{1}{2}\left(n^{2}+3 n\right)-2 ;
$$

but on the ground there explained, the case

$$
\alpha_{1}+3 \alpha_{2}+6 \alpha_{3}+\ldots<\frac{1}{2}\left(n^{2}+3 n\right)-2
$$

is excluded, and we thus have the equation (2), giving with (1) the equation (3). 
I believe the better course is to assume (1) and (3) as the fundamental equations, from them deducing (2); and we thus also get over a difficulty presently referred to, but which did not occur to me when the memoir was written.

In fact, starting with the equations $x^{\prime}: y^{\prime}: z^{\prime}=X: Y: Z$ (which are to give $x: y: z=X^{\prime}: Y^{\prime}: Z^{\prime}$ ), we have in the first instance the equation (1). Moreover, establishing for $x^{\prime}, y^{\prime}, z^{\prime}$ a linear equation $a x^{\prime}+b y^{\prime}+c z^{\prime}=0$, we have corresponding hereto a curve $a X+b Y+c Z=0$, and the coordinates $x, y, z$ of a point on this curve are proportional to $X^{\prime}: Y^{\prime}: Z^{\prime}$; that is, substituting for $z^{\prime}$ the value $-\frac{1}{c}\left(a x^{\prime}+b y^{\prime}\right)$, they are proportional to rational and integral (homogeneous) functions of $\left(x^{\prime}, y^{\prime}\right)$, that is, to rational and integral functions of the single parameter $x^{\prime}: y^{\prime}$; wherefore the curve $a X+b Y+c Z=0$ is unicursal; whence the equation (3). The like change may be made in the theory of the rational transformation between two spaces; and it is in this case a more important one.

The difficulty is as follows: It is not self-evident that we are at liberty to assume

$$
\alpha_{1}+3 \alpha_{2}+6 \alpha_{3} \ldots=\frac{1}{2}\left(n^{2}+3 n\right)-2
$$

for imagine that we had a system of $\left(\alpha_{1}, \alpha_{2}, \alpha_{3}, \ldots\right)$ points, such that $\alpha_{1}+4 \alpha_{2}+\ldots$ being $=n^{2}-1$, and $\alpha_{1}+3 \alpha_{2}+\ldots$ being $>\frac{1}{2}\left(n^{2}+3 n\right)-2$, the points were such that the conditions in question (viz., the condition that the curve passes once through each of the points $\alpha_{1}$, twice through each of the points $\alpha_{2} \ldots$ ! should be less than $\alpha_{1}+3 \alpha_{2}+\ldots$, and in fact $=$ or $<\frac{1}{2}\left(n^{2}+3 n\right)-2$; then the functions $X, Y, Z$ would not of necessity be connected by a linear relation $\lambda X+\mu Y+\nu Z=0$, and the ground for the assumption in question, $\alpha_{1}+3 \alpha_{2}+\ldots \overline{2} \frac{1}{2}\left(n^{2}+3 n\right)-2$, would no longer exist. And except by the process now adopted of deriving the equation (2) from the equations (1) and (3), I do not know how the impossibility of such a system is to be established; viz., I do not know how we are to prove the following theorem:-There is not any system of $\left(\alpha_{1}, \alpha_{2}, \alpha_{3} \ldots\right)$ points, where

$$
\begin{aligned}
& \alpha_{1}+4 \alpha_{2}+9 \alpha_{3} \ldots=n^{2}-1, \\
& \alpha_{1}+3 \alpha_{2}+6 \alpha_{3} \ldots>\frac{1}{2}\left(n^{2}+3 n\right)-2,
\end{aligned}
$$

such that (for a curve of the order $n$ passing once through each point $\alpha_{1}$, twice through each point $\left.\alpha_{2}, \ldots\right)$ the number of conditions actually imposed on the curve is $=$ or $<\frac{1}{2}\left(n^{2}+3 n\right)-2$.

A system of $\left(\alpha_{1}, \alpha_{2} \ldots\right)$ points such that the number of actually imposed conditions is less than $\alpha_{1}+3 \alpha_{2}+\ldots$, may be termed a special system; we have, of course, the well-known case $\left(\alpha_{1}=n^{2}\right)$ of a system of $n^{2}$ points, such that any curve of the order $n$ passing through $\frac{1}{2}\left(n^{2}+3 n\right)-1$ of these passes through all the remaining points for what is the same thing, where the number of conditions actually imposed is $=\frac{1}{2}\left(n^{2}+3 n\right)-1$; ; and we have the following special system, which presented itself to Dr Clebsch, in his researches on the Abbildung of a quintic surface with two non-intersecting nodal 
lines; viz., " $\alpha_{1}=12, \alpha_{2}=2$. We may have 12 points and 2 points such that, for a quintic curve passing once through each of the 12 points and twice through each of the 2 points, the number of conditions actually imposed (instead of being $12+3.2,=18$ ) is = 17." The construction is as follows: viz., starting with the 2 points and any 10 points, we may draw a quartic passing twice through the first of the 2 points, once through the second of them, and through the 10 points; and another quartic passing twice through the second of the 2 points, once through the first of them, and through the 10 points: the two quartics intersect in the 2 points each twice, in the 10 points, and in 2 new points, forming, with the 10 points, a system of 12 points; and the first-mentioned 2 points and the 12 points form the system in question.

A more complicated case, $\alpha_{1}=10, \alpha_{2}=6, \alpha_{3}=1$, occurs in Dr Nöther's paper, "Ueber Flächen, welche Schaaren rationaler Curven besitzen," [Math. Ann., t. III. (1871), pp. 161-227]. Except these two, I do not know any other case of a special system for which $\alpha_{2}, \alpha_{3} \ldots$ are not all $=0$; the investigation of such systems would, I think, be very interesting.

[A concluding paragraph of sevven lines gave some corrections to the "Memoir on the Rational Transformation between Two Spaces," 447, which corrections are made in the present reprint of that paper.] 